\title{
Dissolution/precipitation mechanisms for diagenesis in sedimentary basins
}

\author{
A. C. Fowler \\ Mathematical Institute, University of Oxford, Oxford, UK
}

Xin-She Yang

Faculty of Engineering, University of Wales, Swansea, Singleton Park, Swansea, UK

Received 28 October 2002; revised 16 April 2003; accepted 28 May 2003; published 30 October 2003.

[1] The transformation of hydrated clay minerals (smectite) to anhydrous forms (illite) occurs in the diagenesis of shales in sedimentary basins. The released pore water can lead to excess pore pressures and is of concern in oil drilling operations. Most simply, the process can be modeled as a first-order thermally activated reaction, but an alternative interpretation is that diagenesis occurs via dissolution of smectite in free pore water and subsequent precipitation of illite. We show how such a realistic model of diagenesis can be included in a compactive model of the sediment/pore water system, and by using the limit of weak solubility (also known as solid density asymptotics), we show how an explicit expression can be derived for the smectite dissolution rate, even when this is mediated by feldspar dissolution and other reactions. In certain circumstances, the reaction rate reduces to that of a simple first-order model. By solving the model we also show that shale diagenesis provides a mechanism for the generation of fractures at depth in rapidly deposited sediments. When sedimentation is slow (or compaction is fast), the smectite to illite transition occurs over a reaction window which typically lies below the equilibrated normally pressured part of the profile, but it does not generally contribute significantly to the overpressuring. INDEX TERMS: 1010 Geochemistry: Chemical evolution; 3210 Mathematical Geophysics: Modeling; 8105 Tectonophysics: Continental margins and sedimentary basins (1212); KEYWORDS: dissolution, precipitation, compaction, diagenesis, sedimentary basins

Citation: Fowler, A. C., and X.-S. Yang, Dissolution/precipitation mechanisms for diagenesis in sedimentary basins, J. Geophys. Res., 108(B10), 2509, doi:10.1029/2002JB002269, 2003.

\section{Introduction}

[2] Diagenesis occurs in sedimentary basins when hydrated clay minerals such as smectite are converted to illite by a dehydration reaction. The process is facilitated by high temperatures and pressures, and the resultant water which is released invades the pore space. One consequence of this is that pore water pressures can be increased, thus leading to the phenomenon of over pressuring, which is of concern in oil drilling operations. The purpose of the present paper is to provide a possible model for the mechanism of diagenesis, and in particular to show how a description of the processes of smectite dissolution and quartz precipitation, mediated by the presence of potassium feldspar, can in certain circumstances be effectively considered as occurring via the kinetics of a simple nonlinear reaction scheme.

[3] The conversion of smectite to illite has received a good deal of attention in the literature. Eberl and Hower [1976] essentially treat the reaction using first-order kinetics as

$$
\mathrm{S} \stackrel{k}{\longrightarrow} \mathrm{I},
$$

Copyright 2003 by the American Geophysical Union. 0148-0227/03/2002JB002269\$09.00 where S denotes smectite and I denotes illite, with a thermally activated reaction rate

$$
-\frac{d S}{d t}=k(T) S
$$

where the rate constant $k$ is given by

$$
k=k_{0} \exp [-E / R T] .
$$

Such a description would appear at best to be schematic, insofar as the "reaction" is likely to occur through various subsidiary processes. On the other hand, shale diagenesis does appear to "switch on" quite suddenly at temperatures of about $100^{\circ} \mathrm{C}$ [Eberl and Hower, 1976; Abercrombie et al., 1994], and occurs throughout a diagenetic "window" between about $100^{\circ} \mathrm{C}$ and $200^{\circ} \mathrm{C}$. A thermally activated reaction rate constant such as equation (3) can mimic this behavior if the activation energy $E$ is relatively high. This suggests that simple reaction kinetics may mimic a more realistic scheme. On the other hand, Velde and Vasseur [1992] found that a two-step model was necessary to represent their data. Part of our purpose is to establish conditions under which a detailed dissolution-precipitation process can be represented by a simpler scheme. 
[4] An alternative description to the first-order reaction involves the dissolution of smectite in pore water to form an aqueous silica compound. At the same time, potassium feldspar dissolves to yield potassium (and aluminium hydroxyl) ions in solution. These combine with the aqueous silica compound and precipitate as illite, leaving aqueous silica in solution, which can precipitate as quartz.

[5] A common reaction scheme which includes the role of potassium feldspar is to write

$$
2 \mathrm{~S}+\mathrm{KFs} \rightarrow 2 \mathrm{I}+4 \mathrm{Qz}(\mathrm{aq})+9 \mathrm{H}_{2} \mathrm{O}
$$

(Abercrombie et al. [1994] give the detailed chemistry), where KFs represents potassium feldspar, Qz(aq) is aqueous quartz (i.e., in solution), and $\mathrm{H}_{2} \mathrm{O}$ represents released pore water. Huang et al. [1993] analyzed experimental and field data and derived the reaction rate

$$
-\frac{d S}{d t}=k(T)\left[K^{+}\right] S^{2}
$$

where $\left[K^{+}\right]$is the potassium ion concentration, $S$ is the relative shale fraction of smectite (thus $1-S=I$ is the relative fraction of illite), and $k(T)$ is a temperaturedependent rate constant.

[6] The rate (5) follows from equation (4) by application of the law of mass action but cannot legitimately be derived, since equation (4) is a compound reaction. Our purpose in the remainder of this paper is therefore to pose a model for smectite dissolution and illite precipitation, with a view to establishing conditions under which simplified rates such as equation (5) or (2) may be appropriate. Continuum models of the type we consider below have been presented before [e.g., Steefel and Lasaga, 1994; Aharonov et al., 1997], and applications to the generation of abnormal pore pressures have been made [Wong et al., 1997]. Our approach differs in that we use the weak solubility hypothesis to provide an effective reduction of the governing transport equations to a much simpler form, and we relate the solutions of the resultant model to our earlier work on fast and slow compaction [Fowler and Yang, 1998]. In this way we provide an insight into the potential importance of diagenesis in the generation of high pore pressures.

\section{Precipitation/Dissolution Model of Diagenesis}

[7] The processes of smectite dissolution, illite precipitation, K-feldspar dissolution, and quartz precipitation are written as the following sequence of reactions:

$$
\begin{gathered}
\mathrm{S}^{S} \stackrel{R_{1}}{\longrightarrow} \mathrm{X}^{L}+n \mathrm{H}_{2} \mathrm{O}, \\
\mathrm{KFs} \stackrel{R_{2}}{\longrightarrow} \mathrm{K}^{+L}+\mathrm{Al}(\mathrm{OH})_{4}^{-L}+s \mathrm{SiO}_{2}^{L}, \\
\mathrm{~K}^{+L}+\mathrm{Al}(\mathrm{OH})_{4}^{-L}+f \mathrm{X}^{L} \stackrel{R_{3}}{\longrightarrow} f \mathrm{I}^{S}+\mathrm{SiO}_{2}^{L}, \\
\mathrm{SiO}_{2}^{L} \underset{R_{4}^{-}}{\stackrel{R_{4}^{+}}{\rightleftharpoons}} \mathrm{Qz} .
\end{gathered}
$$

Sequentially, these represent smectite dissolution (superscripts $S, L$ refer to solids or aqueous phases, $\mathrm{S}$ is smectite,
$\mathrm{X}$ is an aqueous silica combination, such as (for example) $\mathrm{Si}_{4} \mathrm{O}_{10}(\mathrm{OH})_{2}$ ); potassium feldspar (KFs) dissolution to form potassium and aluminium hydroxyl ions and aqueous silica $\mathrm{SiO}_{2}$; illite precipitation via the combination of the silica compound with potassium and aluminium hydroxyl ions; and finally the precipitation (or dissolution) of quartz (Qz) from the aqueous silica phase. The coefficients $n, s, f$ are stoichiometric coefficients. The indicated rates $R_{i}$ are the reaction rates of the various reactions.

[8] In order to describe the evolution of the species concentrations, we denote by $S, X, I, K, A, L, Q$ and $F$ the concentrations of smectite, aqueous silica compound, illite, potassium ions, aluminium hydroxyl ions, aqueous silica, quartz, and feldspar. For the solid phases, the units are mol $\mathrm{m}^{-3}$ of solids; for the aqueous phases (assumed dilute), the units are mol m $\mathrm{m}^{-3}$ of pore water. If $\phi$ is the porosity and the pore fluid has velocity $\mathbf{u}^{l}$, the solid matrix has velocity $\mathbf{u}^{s}$ (the same for each solid phase), then conservation laws following from equation (6) are the following:

$$
\begin{gathered}
\frac{\partial}{\partial t}[(1-\phi) S]+\nabla \cdot\left[(1-\phi) S \mathbf{u}^{s}\right]=-r_{1}, \\
\frac{\partial}{\partial t}(\phi X)+\nabla \cdot\left(\phi X \mathbf{u}^{l}\right)=\nabla \cdot(\phi D \nabla X)+r_{1}-f r_{3}, \\
\frac{\partial \phi}{\partial t}+\nabla \cdot\left(\phi \mathbf{u}^{l}\right)=n r_{1} M_{w} / \rho_{w}, \\
\frac{\partial}{\partial t}[(1-\phi) F]+\nabla \cdot\left[(1-\phi) F \mathbf{u}^{s}\right]=-r_{2}, \\
\frac{\partial(\phi K)}{\partial t}+\nabla \cdot\left(\phi K \mathbf{u}^{l}\right)=\nabla \cdot(\phi D \nabla K)+r_{2}-r_{3}, \\
\frac{\partial(\phi A)}{\partial t}+\nabla \cdot\left(\phi A \mathbf{u}^{l}\right)=\nabla \cdot(\phi D \nabla A)+r_{2}-r_{3}, \\
\frac{\partial(\phi L)}{\partial t}+\nabla \cdot\left(\phi L \mathbf{u}^{l}\right)=\nabla \cdot[\phi D \nabla L]+s r_{2}+r_{3}-r_{4}^{+}+r_{4}^{-}, \\
\frac{\partial[(1-\phi) Q]}{\partial t}+\nabla \cdot\left[(1-\phi) Q \mathbf{u}^{s}\right]=r_{4}^{+}-r_{4}^{-} \\
\frac{\partial[(1-\phi) I]}{\partial t}+\nabla \cdot\left[(1-\phi) I \mathbf{u}^{s}\right]=f r_{3},
\end{gathered}
$$

wherein $M_{w}$ is the molecular weight of water and $\rho_{w}$ is its density (thus $\rho_{w} / M_{w}$ is its "concentration" in units of mol $\mathrm{m}^{-3}$ of pore water).

[9] As well as advection, these equations include a diffusion term for the aqueous components (but this is omitted for the water phase due to the diluteness of the solutions). The reaction rates $r_{i}$ correspond to $R_{i}$. If the aqueous phase is assumed well mixed and if precipitation occurs heterogeneously on preexisting solid grains, then

$$
r_{i}=\Sigma_{i} R_{i}
$$

for each $i$, where $\Sigma_{i}$ is the specific interfacial surface area (surface area per volume of rock) of the relevant solid substrate. The units of $R_{i}$ are $\mathrm{mol} \mathrm{s}^{-1}$ (surface area) ${ }^{-1}$, since the dissolution reactions are surface specific; those of $r_{i}$ are mol s $\mathrm{m}^{-1}$ of rock. Prescription of the specific surface areas is hazardous, but the simplest assumption is to write

$$
\Sigma_{1} \propto S ; \Sigma_{2} \propto F ; \Sigma_{3} \propto I ; \Sigma_{4}^{ \pm} \propto Q,
$$

bearing in mind that some adjustment needs to be made for $Q$ and $I$ near zero. In this reaction scheme, we see that 
potassium and aluminium hydroxyl ions act in concert, and we henceforth ignore the hydroxyl ions as separate entities in the discussion.

[10] A general expression for the reaction rates $R_{i}$ is [Lasaga, 1984; Lasaga et al., 1994]

$$
R_{i}=k_{i} f_{i}\left(a_{j}\right) g\left(\Delta G_{i}\right),
$$

where $a_{j}$ are the activities of the species in solution, and a typical assumption is that

$$
f_{i}\left(a_{j}\right)=\prod_{j} a_{j}^{n_{i j}}
$$

where $n_{i j}$ are stoichiometric coefficients of the aqueous species participating in the reaction. The function $g$ depends on the free energy of the solution, where we take $\Delta G_{i}<0$ for undersaturation, and $\Delta G_{i}>0$ for supersaturation. Lasaga et al. [1994] write

$$
g=\left[1-\exp \left(\Delta G_{i} / R T\right)\right]_{+}
$$

for dissolution (where $\left.[x]_{+}=\max (x, 0)\right)$, and

$$
g=\left[\exp \left(\Delta G_{i} / R T\right)-1\right]_{+}
$$

for precipitation. For single species in dilute solutions, we can take $\Delta G=R T \ln \left(c / c_{e q}\right)$, where $c$ is the aqueous concentration, and then

$$
g=\left[1-\left(c / c_{e q}\right)\right]_{+}
$$

for dissolution, and

$$
g=\left[\left(c / c_{e q}\right)-1\right]_{+}
$$

for precipitation, where $c_{e q}$ represents the solubility limit (saturated concentration) of the relevant aqueous species and $[c]_{+}=\max (c, 0)$. More generally, nonlinear expressions of a similar type have also been suggested [e.g., Nielsen, 1964]. The rate coefficients $k_{i}$ will also vary with temperature, in the general Arrhenius form

$$
k_{i}=v_{i} \exp \left[-E_{i} / R T\right],
$$

where $E_{i}$ is the activation energy for the reaction $i$. In experiments [e.g., Eberl and Hower, 1976], the premultiplicative factor is usually given as a frequency (i.e., the reaction rate is given as the frequency

$$
k_{i}^{*}=v_{i}^{*} \exp \left[-E_{i} / R T\right]
$$

since the rate of reaction is measured volumetrically and not surface specifically). The two are related as follows: equation (8) gives the reaction rate in moles per unit volume per unit time. If $M_{i}$ is the molecular weight $\left(\mathrm{kg} \mathrm{mol}^{-1}\right)$ and $\rho_{i}$ the density of the solid substrate $\left(\mathrm{kg} \mathrm{m}^{-3}\right)$, then $M_{i} \Sigma_{i} k_{i} / \rho_{i}$ is the reaction rate as a frequency, i.e., $k^{*}$, and hence

$$
\nu_{i}^{*}=\frac{M_{i} \sum_{i} \nu_{i}}{\rho_{i}} .
$$

[11] It is convenient to write the reaction equations (7) in terms of solid volume fractions. In addition, we will also introduce a convenient scaling for the aqueous concentrations. For a solid concentration $Y^{S}$ (mol m${ }^{-3}$ of solids) of substance $\mathrm{Y}$ with molecular weight $M_{Y}\left(\mathrm{~kg} \mathrm{~mol}^{-1}\right)$ and density $\rho_{Y}\left(\mathrm{~kg} \mathrm{~m}^{-3}\right.$ of rock), $\rho_{Y} / M_{Y}\left(\mathrm{~mol} \mathrm{~m}{ }^{-3}\right.$ of $\left.\mathrm{Y}\right)$ is the molar density of $Y$, thus $(1-\phi) Y^{S} M_{Y} / \rho_{Y}$ is the quantity of $Y$ $\left(\mathrm{m}^{3}\right.$ of $\mathrm{Y} \mathrm{m}^{-3}$ of rock), and is thus the volume fraction $\phi_{Y}$ of Y. Therefore we define (writing $Y^{S}=Y$ )

$$
\phi_{Y}=\frac{(1-\phi) Y M_{Y}}{\rho_{Y}}
$$

for $\mathrm{Y}=\mathrm{S}, \mathrm{F}, \mathrm{Q}, \mathrm{I}$. We scale the aqueous concentrations $X, L$, $K$ in terms of their solubility limits $c_{X S}$ (aqueous silica compound in the presence of smectite), $c_{L Q}$ (aqueous silica in the presence of quartz) and $c_{K F}$ (potassium ions in the presence of feldspar); these may be given in dimensionless units as parts per million (by weight: $1 \mathrm{ppm}=1 \mathrm{mg}$ solute per $1 \mathrm{~kg}$ solution). For example, the solubility of quartz takes a value of about $6 \mathrm{ppm}$ at room temperature (the activity is about $10^{-4} \mathrm{M}$, and the gram molecular weight of $\mathrm{SiO}_{2}$ is 60) [Krauskopf and Bird, 1995], while that of an amorphous silica phase is higher, about $120 \mathrm{ppm}$. These values increase with temperature, by factors of about 3 to 5 at $100^{\circ} \mathrm{C}$ [Krauskopf and Bird, 1995]. In our model, we associate these two solubility limits with illite precipitation and smectite precipitation in the presence of feldspar, i.e., $c_{X I}$ and $c_{X S}$ [Aagaard and Helgeson, 1983]; more generally, all these solubility limits are very small [Rimstidt, 1997]. The dissolved species $\mathrm{D}^{L}(\mathrm{D}=\mathrm{X}, \mathrm{L}$ or $\mathrm{K})$ has concentration $D^{L}$, with units of $\mathrm{mol} \mathrm{m} \mathrm{m}^{-3}$ of pore water. If $\mathrm{D}^{L}$ has molecular weight $M_{D}\left(\mathrm{~kg} \mathrm{~mol}^{-1}\right)$, then $M_{D} D^{L}$ is the density in $\mathrm{kg} \mathrm{m}^{-3}$. A convenient normalization is to write this in terms of the solubility limit. If this is $c_{D}$ ppm, we write $c_{D}^{*}=$ $c_{D} \times 10^{-6}$, and then we define the dimensionless concentration of $\mathrm{D}^{L}, \psi_{D}$, via

$$
D^{L}=\frac{\rho_{w}}{M_{D}} c_{D}^{*} \psi_{D}
$$

so that $\psi_{D}$ is the density of $\mathrm{D}^{L}$ relative to that at saturation. Values of $\psi_{D}>1$ correspond to supersaturated pore water, whereas values $<1$ represent unsaturated pore water.

[12] Equations (7) can now be written in the form

$$
\begin{aligned}
& \frac{\partial \phi_{S}}{\partial t}+\nabla \cdot\left[\phi_{S} \mathbf{u}^{s}\right]=-\frac{M_{S}}{\rho_{S}} r_{1}, \\
& \frac{\partial \phi_{F}}{\partial t}+\nabla \cdot\left[\phi_{F} \mathbf{u}^{s}\right]=-\frac{M_{F}}{\rho_{F}} r_{2},
\end{aligned}
$$

$$
\frac{\partial \phi_{Q}}{\partial t}+\nabla \cdot\left[\phi_{Q} \mathbf{u}^{s}\right]=\frac{M_{Q}}{\rho_{Q}}\left(r_{4}^{+}-r_{4}^{-}\right)
$$

$$
\frac{\partial \phi_{I}}{\partial t}+\nabla \cdot\left[\phi_{I} \mathbf{u}^{s}\right]=f \frac{M_{I}}{\rho_{I}} r_{3}
$$

$$
\frac{\partial \phi}{\partial t}+\nabla \cdot\left(\phi \mathbf{u}^{l}\right)=n \frac{M_{w}}{\rho_{w}} r_{1}
$$




$$
\begin{aligned}
& c_{X S}^{*}\left[\frac{\partial}{\partial t}\left(\phi \psi_{X}\right)+\nabla \cdot\left[\phi \psi_{X} \mathbf{u}^{l}\right]-\nabla \cdot\left[\phi D \nabla \psi_{X}\right]\right]=\frac{M_{X}}{\rho_{w}}\left(r_{1}-f r_{3}\right), \\
& c_{K F}^{*}\left[\frac{\partial}{\partial t}\left(\phi \psi_{K}\right)+\nabla \cdot\left[\phi \psi_{K} \mathbf{u}^{l}\right]-\nabla \cdot\left[\phi D \nabla \psi_{K}\right]\right]=\frac{M_{K}}{\rho_{w}}\left(r_{2}-r_{3}\right),
\end{aligned}
$$

$$
\begin{aligned}
& c_{L Q}^{*}\left[\frac{\partial}{\partial t}\left(\phi \psi_{L}\right)+\nabla \cdot\left[\phi \psi_{L} \mathbf{u}^{l}\right]-\nabla \cdot\left[\phi D \nabla \psi_{L}\right]\right] \\
& \quad=\frac{M_{L}}{\rho_{w}}\left\{s r_{2}+r_{3}-\left(r_{4}^{+}-r_{4}^{-}\right)\right\} ;
\end{aligned}
$$

Equations (20) form the basis of our subsequent discussion.

\section{Weak Solubility and the Pseudo-Steady State Hypothesis}

[13] We begin our discussion by noting that the total volume fraction of solids and liquid must be conserved; if $S$, $Q, F$, and $I$ constitute all the solid mass, then (ignoring the small quantities of dissolved species) we have

$$
\phi+\phi_{S}+\phi_{F}+\phi_{Q}+\phi_{I}=1 ;
$$

if there is another (unreactive) solid fraction $\phi_{U}=1-(\phi+$ $\left.\phi_{S}+\phi_{F}+\phi_{Q}+\phi_{I}\right)$, then also $\partial \phi_{U} / \partial t+\nabla \cdot\left[\phi_{U} \mathbf{u}^{s}\right]=0$. This extra equation is necessary when the present diagenesis theory is subsumed into a compaction model.

[14] Our principal aim in this section is to relate the model incorporated in Equations (20) to those of other, simpler models. These can be represented as the following reaction schemes, respectively.

[15] Scheme I is a one-step (dehydration) reaction:

$$
\mathrm{S}^{S} \rightarrow \mathrm{I}^{S}+n \mathrm{H}_{2} \mathrm{O} .
$$

The smectite-illite transformation is considered to be a first order reaction through the dehydration of smectite [Eberl and Hower, 1976; Huang et al., 1993; Abercrombie et al., 1994].

[16] Scheme II is a two-step precipitation/dissolution reaction:

$$
\begin{gathered}
\mathrm{S}^{S} \rightarrow \mathrm{X}^{L}+n \mathrm{H}_{2} \mathrm{O}, \\
\mathrm{X}^{L} \rightarrow \mathrm{I}^{S} .
\end{gathered}
$$

The intermediate aqueous silica combination is included, but the mediating role of $\mathrm{K}$-feldspar is neglected. Reactions of this type have implicitly been considered by Lasaga [1981] and Velde and Vasseur [1992].

\subsection{Kinetics Without Transport}

[17] The basic simplification we can make to the complete reaction model we have posed can be called the "weak solubility limit"; it is analogous to the pseudo-steady state hypothesis in enzyme kinetics and is due to the fact that the solubility limits $c_{D Y}^{*}$ in Equations (20) are all extremely small [Rimstidt, 1997]. In the context of geochemically reactive flows in porous media, the same approximation is known as "solid density asymptotics" [Ortoleva, 1994]. For example, using the values for $c_{X S}$ and $c_{X I}$ of $120 \mathrm{ppm}$ and $6 \mathrm{ppm}$ quoted previously [Aagaard and Helgeson, 1983], we have $c_{X S}^{*}=120 \times 10^{-6}, c_{X I}^{*}=6 \times 10^{-6}$. On the assumption that $c_{X S}^{*}, c_{L Q}^{*}, c_{K F}^{*} \ll 1$, we can assume the corresponding reactions $(20 \mathrm{f}),(20 \mathrm{~g})$, and $(20 \mathrm{~h})$ are at equilibrium, whence there follows

$$
\begin{aligned}
r_{1} & \approx f r_{3}, \\
r_{2} & \approx r_{3}, \\
r_{4}^{+}-r_{4}^{-} & \approx(s+1) r_{3} .
\end{aligned}
$$

Substitution into the equations for $\phi_{J}$ then gives equations for the solid concentrations; however, the on-off nature of the precipitation/dissolution kinetics makes the procedure a little more subtle.

[18] To see this, we adopt specific forms for the reaction rates based on the prescription in section 2. Equations (8), (10), (11), and (14) give the reaction rates in the general form ( $D$ for dissolution, $P$ for precipitation)

$$
\begin{aligned}
& r_{i}^{D}=\Sigma_{i} k_{i} f_{i}\left[1-\frac{c_{i}}{c_{q}}\right]_{+}, \\
& r_{i}^{P}=\Sigma_{i} k_{i} f_{i}\left[\frac{c_{i}}{c_{q}}-1\right]_{+},
\end{aligned}
$$

where $c_{i}$ is the relevant aqueous concentration and $c_{q}$ the associated solubility limit. $\Sigma_{i}$ is the precipitate surface area, $k_{i}$ is the thermally activated rate constant, and $f_{i}$ is the activity dependence: we leave these as fixed for the moment. In consideration of equation (6), we have to generalize equation (25) to accommodate precipitation or dissolution of two solutes.

[19] We do this in the obvious way, allowing products of the excess concentration factors such as $\left[c_{i} / c_{q}-1\right]_{+}$. Specifically, reactions two and three in equation (6) represent dissolution of feldspar and precipitation of illite, and are controlled respectively by the liquid phases $\mathrm{K}^{+L}$ and $\mathrm{SiO}_{2}^{L}$ (for equation (6b)) and $\mathrm{K}^{+L}$ and $\mathrm{X}^{L}$ (for equation (6c)). Thus we assume that dissolution in these two reactions is controlled by the product of the appropriate concentration factors, and when written in terms of the dimensionless aqueous concentrations $\psi_{D}(D=L, K, X)$, we find that equation (6c) can be written in the form

$$
\begin{aligned}
r_{1}^{D} & =\Sigma_{S} k_{1} f_{1}\left[1-\psi_{X}\right]_{+}, \\
r_{2}^{D} & =\Sigma_{f} k_{2} f_{2}\left[1-\psi_{K}\right]_{+}\left[\theta_{L}-\psi_{L}\right]_{+}, \\
r_{3}^{P} & =\Sigma_{I} k_{3} f_{3}\left[\psi_{K}-\theta_{K}\right]_{+}\left[\psi_{X}-\theta_{X}\right]_{+}, \\
r_{4}^{P} & =\Sigma_{Q} k_{4}^{+} f_{4}^{+}\left[\psi_{L}-1\right]_{+}, \\
r_{4}^{D} & =\Sigma_{Q} k_{4}^{-} f_{4}^{-}\left[1-\psi_{L}\right]_{+},
\end{aligned}
$$

where the solubility ratios are defined as

$$
\begin{aligned}
& \theta_{L}=c_{L F} / c_{L Q}, \\
& \theta_{K}=c_{K I} / c_{K F}, \\
& \theta_{X}=c_{X I} / c_{X S} .
\end{aligned}
$$

[20] Various possibilities now occur depending on the values of the different solubility limits. We have indicated 
that silica compound $(X)$ solubility with respect to illite is much less than that with respect to smectite [Aagard and Helgeson, 1983; Sass et al., 1987] (thus $\theta_{X} \ll 1$ ), and we suppose this is also true for potassium, that is to say $\theta_{K} \ll 1$ [Aagard and Helgeson, 1983, Figure 14]. On the other hand, we suppose that the solubilities of silica with respect to feldspar or quartz are comparable, so that $\theta_{L} \sim 1$. We denote the reaction terms $k_{i} f_{i}$ in equation (26) as

$$
k_{i} f_{i}=\bar{R} \mathcal{R}_{i}
$$

where $\bar{R}$ (mol area ${ }^{-1}$ time $^{-1}$ ) is a normalization to be defined below (and $\mathcal{R}_{i}$ is dimensionless) and we specifically write, for notational convenience,

Smectite dissolution Feldspar dissolution Illite precipitation Quartz precipitation Quartz dissolution

$$
\begin{aligned}
& \mathcal{R}_{1}=\mathcal{R}_{S D}, \\
& \mathcal{R}_{2}=\mathcal{R}_{F D}, \\
& \mathcal{R}_{3}=\mathcal{R}_{I P}, \\
& \mathcal{R}_{4}^{+}=\mathcal{R}_{Q P}, \\
& \mathcal{R}_{4}^{-}=\mathcal{R}_{Q D},
\end{aligned}
$$

bearing in mind that $\mathcal{R}_{i}$ may typically depend (perhaps algebraically) on concentrations (via $f_{i}$ ) and on temperature (via $k_{i}$ ). For simplicity, we suppress the dependence of $\mathcal{R}_{i}$ on aqueous phase concentrations. The typical form for the reaction rates is then

$$
\mathcal{R}_{i}=\exp \left[\lambda_{i}\left(T-T_{i}\right)\right]
$$

equation (30) represents a Frank-Kamenetskii [1955] approximation to the Arrhenius rate law; $T_{i}$ is a characteristic "switch-on" temperature, and $\lambda_{i}$ is proportional to the activation energy $E_{i}$. Specifically, if the dimensional reaction rate is $k_{i}=v_{i} \exp \left(-E_{i} / R T\right)$, then the FrankKamenetskii approximation is that $k_{i}=\bar{R} \mathcal{R}_{i}$, where $\mathcal{R}_{i}$ is given by equation (30), and

$$
T_{i}=\frac{\left(E_{i} / R\right)}{\ln \left(\nu_{i} / \bar{R}\right)}, \quad \lambda_{i}=E_{i} / R T_{i}^{2} .
$$

It is valid in practice if $T_{i} \ll E / R$, or equivalently $\nu_{i} \gg \bar{R}$, which we shall later find to be an accurate assumption. Eberl and Hower [1976] report experimental results from which we can infer values $E_{I P} \approx 80 \mathrm{~kJ} \mathrm{~mol}^{-1}$ and $\nu_{I P} * \approx 1.3 \mathrm{~s}^{-1}$ (see equation (17)). Other estimates of the activation energy are similar to this; for example, Lasaga [1984] quotes a range 40-80 $\mathrm{kJ} \mathrm{mol}^{-1}$ for other mineral reactions, while Rimstidt and Barnes [1980] quote dissolution values of $50 \mathrm{~kJ} \mathrm{~mol}^{-1}$ and precipitation values in the range $67-76 \mathrm{~kJ} \mathrm{~mol}^{-1}$ for a simple silica-water reaction. Huang et al. [1993] find a higher activation energy (about $120 \mathrm{~kJ} \mathrm{~mol}^{-1}$ ) with an appropriately lower $\nu^{*}$ (about $0.4 \times 10^{-5} \mathrm{~s}^{-1}$ ), but these data are from experiments at higher temperatures, above $250^{\circ} \mathrm{C}$.

[21] Indirect inferences of $T_{i}$ in equation (31) come from measured illite fraction in illite/smectite in deep wells. Many different wells show that the smectite-illite reaction starts at typical temperatures which range from about $70^{\circ}$ to about $120^{\circ}$ [Abercrombie et al., 1994; Freed and Peacor, 1989; Pearson and Small, 1988], and the reaction occurs over a temperature range upward of $20^{\circ}$, and over a corresponding depth interval of $0.5-1 \mathrm{~km}$, beginning at depths between 1.5 and $3 \mathrm{~km}$ [Abercrombie et al., 1994;
Freed and Peacor, 1989; Pearson and Small, 1988; Hower et al., 1976]. We use these field and experimental observations later to estimate reasonable values of $T_{i}$ and $\lambda_{i}$.

[22] The equilibrium equations (24) are then (with $\left.r_{3} / \bar{R}=\bar{r}\right)$

$$
\begin{gathered}
\Sigma_{Q}\left[\mathcal{R}_{4}^{+}\left(\psi_{L}-1\right)_{+}-\mathcal{R}_{4}^{-}\left(1-\psi_{L}\right)_{+}\right]=(s+1) \bar{r}, \\
\Sigma_{S} \mathcal{R}_{1}\left(1-\psi_{X}\right)_{+}=f \bar{r}, \\
\Sigma_{F} \mathcal{R}_{2}\left(1-\psi_{X}\right)_{+}\left(\theta_{L}-\psi_{L}\right)_{+}=\bar{r}, \\
\bar{r}=\Sigma_{I} \mathcal{R}_{3}\left(\psi_{K}-\theta_{K}\right)_{+}\left(\psi_{X}-\theta_{X}\right)_{+},
\end{gathered}
$$

and these are four equations for the reaction rate $\bar{r}$ and the three aqueous concentrations $\psi_{X}, \psi_{L}$, and $\psi_{K}$. Assuming nonzero reaction rates, and taking $\theta_{K}, \theta_{X} \ll$ 1 , and $\theta_{L}>1$ (see discussion after equation (27)), we deduce from equation (32) that $\psi_{L}>1$ (thus quartz precipitates from solution), and

$$
\begin{aligned}
\bar{r} & =\Sigma_{I} \mathcal{R}_{3} \psi_{K} \psi_{X}, \\
\psi_{X} & =1-\frac{f \bar{r}}{\Sigma_{S} \mathcal{R}_{1}}, \\
\psi_{L} & =1+\frac{(s+1) \bar{r}}{\Sigma_{Q} \mathcal{R}_{4}^{+}}, \\
\psi_{K} & =1-\frac{\bar{r}}{\Sigma_{F} \mathcal{R}_{2}\left\{\theta_{L}-1-\frac{(s+1) \mathcal{R}_{3}}{\Sigma_{Q} \mathcal{R}_{4}^{+}}\right\}},
\end{aligned}
$$

whence the basic reaction rate $r_{3}$ is given by

$$
r_{3}=\bar{R} \bar{r}=\left(\bar{R} / d_{g}\right) \phi_{I} \mathcal{R}_{I P} r^{*},
$$

where

$$
r^{*} \approx\left[1-\left(\frac{f \phi_{I} \mathcal{R}_{I P}}{\phi_{S} \mathcal{R}_{S D}}\right) r^{*}\right]\left[1-\frac{\left(\phi_{I} \mathcal{R}_{I P} / \phi_{F} \mathcal{R}_{F D}\right) r^{*}}{\left\{\theta_{L}-1-\left(\frac{(s+1) \phi_{I} \mathcal{R}_{I P}}{\phi_{Q} \mathcal{R}_{Q P}}\right) r^{*}\right\}}\right],
$$

and we have assumed that the specific mineral surface areas $\Sigma_{i}$ are given by

$$
\Sigma_{i}=\phi_{i} / d_{g}
$$

where $d_{g}$ is a measure of grain size. Equation (35) determines $r^{*}$ uniquely (taking into account the constraints that $\psi_{X}, \psi_{K}>0$, and hence (since $\bar{r}>0$ ) are also $<1$ ) as a function of the relative reaction rates $\mathcal{R}_{I P} / \mathcal{R}_{S D}, \mathcal{R}_{I P} / \mathcal{R}_{F D}$, $\mathcal{R}_{I P} / \mathcal{R}_{Q P}$, i.e., the rate of illite precipitation as compared to those of smectite dissolution, feldspar dissolution and quartz precipitation. It can in fact be written explicitly as the solution of a quadratic equation, providing $\theta_{L}>1$, i.e., $c_{L F}>$ $\mathrm{c}_{L Q}$. If $\theta_{L} \rightarrow 1+$, then $r^{*} \rightarrow 0$, and if $\theta_{L} \leq 1$, then inspection of equation (32) shows that $r_{3}=0$, and hence also

$$
\psi_{L}=1, \psi_{X}=1, \psi_{K}=\theta_{K}
$$

the aqueous phases rapidly reach saturation, and the smectite-illite transition is unable to proceed. However, it 
is typically the case that $\theta_{L}>1$ [Aagard and Helgeson, 1983].

[23] Substitution of the reaction rates given by equations (24) into the mineral phase conservation equations gives

$$
\begin{gathered}
\frac{\partial \phi_{S}}{\partial t}+\nabla \cdot\left[\phi_{S} \mathbf{u}^{s}\right]=-\left(f \frac{M_{S}}{\rho_{S}}\right) r_{3}, \\
\frac{\partial \phi_{F}}{\partial t}+\nabla \cdot\left[\phi_{F} \mathbf{u}^{s}\right]=-\left(\frac{M_{F}}{\rho_{F}}\right) r_{3}, \\
\frac{\partial \phi_{Q}}{\partial t}+\nabla \cdot\left[\phi_{Q} \mathbf{u}^{s}\right]=\left\{(s+1) \frac{M_{Q}}{\rho_{Q}}\right\} r_{3}, \\
\frac{\partial \phi_{I}}{\partial t}+\nabla \cdot\left[\phi_{I} \mathbf{u}^{s}\right]=\left(f \frac{M_{I}}{\rho_{I}}\right) r_{3}, \\
\frac{\partial \phi}{\partial t}+\nabla \cdot\left[\phi \mathbf{u}^{l}\right]=\left(n f \frac{M_{w}}{\rho_{w}}\right) r_{3} .
\end{gathered}
$$

[24] Summation of the solid phase equations (together with an inert phase, if one exists) also gives

$$
-\frac{\partial \phi}{\partial t}+\nabla \cdot\left[(1-\phi) \mathbf{u}^{s}\right]=\left[\frac{f M_{I}}{\rho_{I}}+(s+1) \frac{M_{Q}}{\rho_{Q}}-\frac{M_{F}}{\rho_{F}}-\frac{f M_{S}}{\rho_{S}}\right] r_{3},
$$

but in general (even with the simplifications we have introduced), the rate $r_{3}$ is a complicated function of the individual components of the solid phases. Note also that if we add equations (38e) and (39), we have

$\boldsymbol{\nabla} \cdot\left[(1-\phi) \mathbf{u}^{s}+\phi \mathbf{u}^{l}\right]=\left[\frac{f M_{I}}{\rho_{I}}+(s+1) \frac{M_{Q}}{\rho_{Q}}-\frac{M_{F}}{\rho_{F}}-\frac{f M_{S}}{\rho_{S}}+\left(n f \frac{M_{w}}{\rho_{w}}\right)\right] r_{3}$,

so that if the diagenetic reactions are significant, then in general the mixture is not incompressible.

[25] It is convenient to write these equations in dimensionless form. We let $d$ denote a physically relevant depth scale, typically several kilometers, over which porosity changes due to mechanical compaction operate, for example. The sedimentation rate $\dot{m}$ defines a natural velocity scale for $\mathbf{u}^{s}$ and $\mathbf{u}^{l}$, and thus $d / \dot{m}$ defines a natural burial timescale. Writing $t, \mathbf{x}$ and the velocities in terms of these, we then obtain the dimensionless set

$$
\begin{aligned}
\frac{\partial \phi_{S}}{\partial t}+\nabla \cdot\left[\phi_{S} \mathbf{u}^{s}\right] & =-R^{*}, \\
\frac{\partial \phi_{F}}{\partial t}+\nabla \cdot\left[\phi_{F} \mathbf{u}^{s}\right] & =-\alpha_{F} R^{*}, \\
\frac{\partial \phi_{Q}}{\partial t}+\nabla \cdot\left[\phi_{Q} \mathbf{u}^{s}\right] & =-\alpha_{Q} R^{*}, \\
\frac{\partial \phi_{I}}{\partial t}+\nabla \cdot\left[\phi_{I} \mathbf{u}^{s}\right] & =\alpha_{I} R^{*}, \\
\frac{\partial \phi}{\partial t}+\nabla \cdot\left[\phi \mathbf{u}^{l}\right] & =\alpha_{w} R^{*},
\end{aligned}
$$

where

$$
R^{*}=f \phi_{I} \mathcal{R}_{I P} r^{*}
$$

and we have finally chosen the reaction rate normalization $\bar{R}$ (see equation (28)) as

$$
\bar{R}=\frac{\dot{m} d_{g} \rho_{S}}{d M_{S}} .
$$

The molar density ratios are given by

$$
\alpha_{F}=\frac{\rho_{S} M_{F}}{f \rho_{F} M_{S}}, \alpha_{Q}=\frac{(s+1) \rho_{S} M_{Q}}{f \rho_{Q} M_{S}}, \alpha_{I}=\frac{\rho_{S} M_{I}}{\rho_{I} M_{S}}, \alpha_{w}=\frac{n \rho_{S} M_{w}}{\rho_{w} M_{S}} .
$$

We assume the following values of the molecular weights, densities and stoichiometric constants (using units of $\mathrm{g} \mathrm{cm}^{-3}$ for density and $\mathrm{g} \mathrm{mol}^{-1}$ for molecular weight, and following chemical formulae of Abercrombie et al. [1994]):

$$
\begin{gathered}
\rho_{w}=1, \quad \rho_{Q} \approx \rho_{F} \approx \rho_{S} \approx \rho_{I} \approx 2.6, \\
M_{S}=450.2, \quad M_{I}=388.2, \\
M_{F}=278.3, \quad M_{w}=18, \quad M_{Q}=60, \\
f=2, \quad s=3, \quad n=4.5,
\end{gathered}
$$

whence values of $\alpha_{i}$ are given by

$$
\alpha_{F}=0.31, \quad \alpha_{Q}=0.27, \quad \alpha_{I}=0.86, \quad \alpha_{w}=0.47 .
$$

We assume typical values of $\dot{m}, d_{g}$ and $d$ to be

$$
\dot{m}=300 \mathrm{~m} \mathrm{Myr}^{-1}=10^{-11} \mathrm{~m} \mathrm{~s}^{-1}, d=1000 \mathrm{~m}, \quad d_{g}=10^{-4} \mathrm{~m}
$$

We then find that the reaction rate normalization parameter $\bar{R} \approx 0.6 \times 10^{-18} \mathrm{~g} \mathrm{~mol} \mathrm{~cm}^{-2} \mathrm{~s}^{-1}$.

\subsection{Simplifications}

[26] The dimensionless smectite reaction rate $R^{*}$ is given, from equations (42) and (35), by

$$
R^{*} \approx f \phi_{I} \mathcal{R}_{I P}\left[1-\frac{R^{*}}{\phi_{S} \mathcal{R}_{S D}}\right]\left[1-\frac{R^{*}}{\phi_{F} \mathcal{R}_{F D}\left\{f\left(\theta_{L}-1\right)-\frac{(s+1) R^{*}}{\phi_{Q} \mathcal{R}_{Q P}}\right\}}\right],
$$

and thus is a function of all four solid fractions $\phi_{I}, \phi_{S}, \phi_{F}$ and $\phi_{Q}$. It is uniquely determined since, although equation (48) has two positive roots, only the smaller of these has physical meaning, for the reasons stated following equation (36). In general, therefore, all the mineral phase equations need to be solved separately. In practice, it is of interest to see whether any simplification is possible if any of the 
dissolution or precipitation reactions are rate limiting, and in fact, this is the case for illite precipitation. Quartz precipitation is slow [Abercrombie et al., 1994; Bjørlykke and Egeberg, 1993], and feldspar dissolution is fast [Busenberg and Clemency, 1976; Gautier et al., 1994]. If we suppose that $\mathcal{R}_{Q P} \ll 1$ and $\mathcal{R}_{F D} \ll \mathcal{R}_{Q P}$ (Bjørlykke and Egeberg [1993] infer values $\mathcal{R}_{Q P} \sim 10^{-5}$, Gautier et al. [1994] imply $\mathcal{R}_{F D} \sim 10$ ), then the last bracketed term in equation (48) is approximately one, and thus equation (48) simplifies to

$$
\frac{1}{R^{*}} \approx \frac{1}{f \phi_{I} \mathcal{R}_{I P}}+\frac{1}{\phi_{S} \mathcal{R}_{S D}}
$$

[27] The reaction rates of smectite and illite, $\mathcal{R}_{S D}$ and $\mathcal{R}_{I P}$, are given by $\mathcal{R}_{i}=\exp \left[\lambda_{i}\left(T-T_{i}\right)\right]$. Values of $T_{i}$ and $\lambda_{i}$ are determined by equation (31). Using equations (17), (36), and (43), we can write

$$
\frac{\nu_{S}}{\bar{R}}=\nu_{S}^{*} t_{b},
$$

where

$$
t_{b}=\frac{d}{\dot{m} \phi_{S}}
$$

is a representative burial time to the depth $d$. We choose values $d=1000 \mathrm{~m}$ and $\dot{m}=300 \mathrm{~m} \mathrm{Myr}^{-1}=10^{-11} \mathrm{~s}^{-1}$ so that $t_{b} \sim 10^{14} \mathrm{~s}$. If we use Eberl and Hower's [1976] data $E_{I P}=80 \mathrm{~kJ} \mathrm{~mol}^{-1}, \nu_{I P}^{*}=1.3 \mathrm{~s}^{-1}$, and suppose that the equivalent of equation (50) applies, then $\nu_{I P} / \bar{R} \sim 10^{14}$ (and is indeed large as we supposed), and we find $T_{I P} \sim 300 \mathrm{~K}$, or about $30^{\circ}$. This is actually quite close to the observed onset reaction temperatures of $70-120^{\circ}(340-400 \mathrm{~K})$ (they appear worse in the arbitrarily zeroed centigrade scale). The value of $T_{I P}$ is very sensitive to the values of $E_{I P}$ and $\nu_{I P}^{*}$; for example if we use Huang et al.'s [1993] values of $120 \mathrm{~kJ}$ $\mathrm{mol}^{-1}$ and $10^{-5} \mathrm{~s}^{-1}$, we find $T_{I P}=700 \mathrm{~K}$, which is clearly inappropriate. Our preferred choice of $T_{I P}$ and also $T_{S D}$ is thus $\approx 100^{\circ} \mathrm{C}$, and we denote this common value as $T_{c}$. The value of $E_{I P}=80 \mathrm{~kJ} \mathrm{~mol}^{-1}$ then implies $\lambda_{I P} \approx 0.07 \mathrm{~K}^{-1}$. Following Rimstidt and Barnes [1980], we might expect that $E_{I P}>E_{S D}$, and thus $\lambda_{I P}>\lambda_{S D}$. In this case $\mathcal{R}_{S D}>\mathcal{R}_{I P}$ for $T<T_{c} \approx 100^{\circ} \mathrm{C}$, while $\mathcal{R}_{S D}<\mathcal{R}_{I P}$ for $T>T_{c}$. Approximately,

$$
\begin{array}{ll}
R^{*} \approx f \phi_{I} \mathcal{R}_{I P}, & T<T_{c}, \\
R^{*} \approx \phi_{S} \mathcal{R}_{S D}, & T>T_{c} .
\end{array}
$$

The reaction would then be rate limited by illite precipitation for $T<T_{c}$ but by smectite dissolution for $T>T_{c}$, and the effect of equation (52) is to slow the reaction to that of the rate-limiting precipitation/dissolution process. The (apparently unlikely) case where $f \mathcal{R}_{S D} \ll \mathcal{R}_{I P}$ reproduces firstorder kinetics precisely, with

$$
R^{*} \approx \mathcal{R}_{S I} \phi_{S}
$$

where

$$
\mathcal{R}_{S I}=f \mathcal{R}_{S D},
$$

but this may not be quantitatively accurate in practice. Note that equation (53) is precisely what one obtains from reaction (22) (or reaction (23) using the weak solubility limit) if the scaled smectite to illite reaction rate is $\mathcal{R}_{S I}$.

[28] The simplification which leads us to equation (49) allows the model to be reduced to three equations for $\phi, \phi_{S}$ and $\phi_{I}$, and this is detailed in section 4; this is because the reduced reaction rate $R^{*}$ in equation (49) is $R^{*}\left(\phi_{S}, \phi_{I}\right)$. In fact, inspection of equation (41) shows that the subsidiary mineral phase concentrations $\phi_{F}$ and $\phi_{O}$ can be written explicitly as linear combinations of $\phi_{S}$ and $\phi_{I}$; therefore this reduction works in general. To be specific, suppose that the solid phase $\mathrm{Y}$ with volume fraction $\phi_{Y}$ satisfies

$$
\frac{\partial \phi_{Y}}{\partial t}+\nabla \cdot\left[\phi_{Y} \mathbf{u}^{s}\right]=\alpha_{Y} R^{*}
$$

together with the boundary condition

$$
\phi_{Y}=\phi_{Y}^{0} \quad \text { on } \quad \partial V
$$

where $\partial V$ denotes the top surface. Then $\phi_{Y}$ is a simple linear combination of $\phi_{I}$ and $\phi_{S}$, explicitly

$$
\phi_{Y}=\frac{\left(\alpha_{Y} \phi_{S}^{0}+\phi_{Y}^{0}\right) \phi_{I}+\left(\alpha_{I} \phi_{Y}^{0}-\alpha_{Y} \phi_{I}^{0}\right) \phi_{S}}{\alpha_{I} \phi_{S}^{0}+\phi_{I}^{0}} .
$$

\section{One-Dimensional Model}

[29] Let us suppose that the smectite reaction rate $R^{*}$ can be taken as a function of $\phi_{I}$ and $\phi_{S}$ only. (At least in the present reaction scheme, this can always be done using equation (57).) We construct a one-dimensional model by adding to the mass conservation equations in equation (41) the equation of momentum conservation in the form of Darcy's law, together with constitutive laws for the effective pressure and the permeability. The details of the equations and their scaling have been given several times previously [Audet and Fowler, 1992; Fowler and Yang, 1998, 1999] and are therefore only summarized here. The generalized form of Darcy's law written in nondimensional form (for a one-dimensional flow) is

$$
\phi\left(u^{l}-u^{s}\right)=\lambda \tilde{k}\left(\frac{\partial \tilde{p}}{\partial z}+1-\phi\right)
$$

where

$$
\lambda=\frac{k_{0}\left(\rho_{s}-\rho_{l}\right) g}{\mu \dot{m}_{s}^{0}}
$$

is a dimensionless parameter measuring the rate of compaction; $k_{0}$ is the uncompacted permeability, $\rho_{s}$ and $\rho_{l}$ are solid and liquid densities (we assume all the solid phases have the same density), $\mu$ is the pore water viscosity, $g$ is gravity, and $\dot{m}_{s}{ }^{0}$ is the specific choice of sedimentation rate scale. Large values of $\lambda$ correspond to rapid compaction or slow sedimentation, while small values indicate slow compaction or rapid accumulation. Both small and large values are possible, although large values are likely to be 

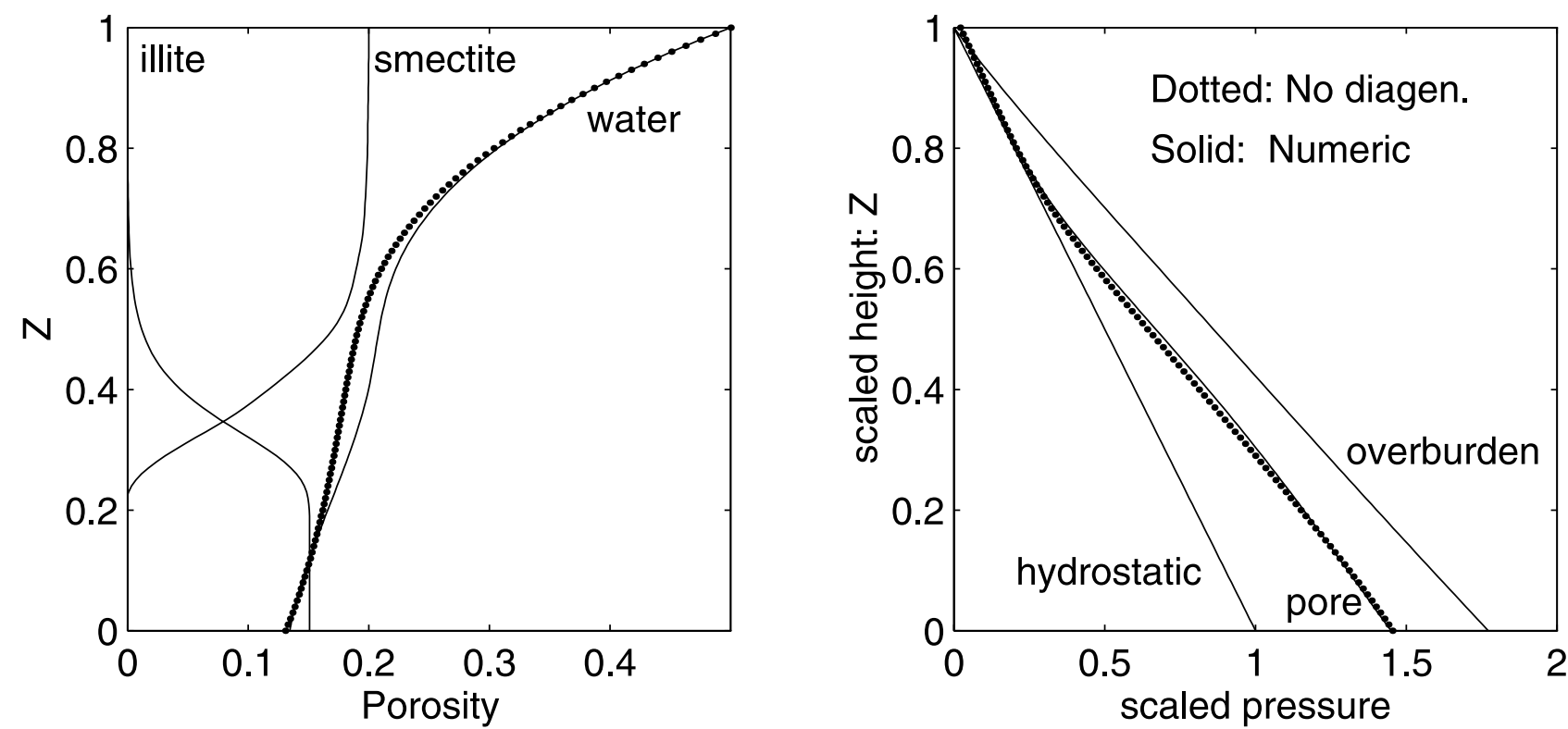

Figure 1. Fast compaction with $\lambda=100$, and the same first-order kinetics as in Figure 2. Snapshots of porosity and pressure profiles in terms of scaled height $Z=z / h(t)$ at $t=5$. The dotted curve corresponds to the absence of diagenesis, that is $\alpha_{I}=1, \alpha_{w}=0$. The diagenetic case corresponds to values $\alpha_{I}=0.86, \alpha_{w}=$ 0.47 . The effect on porosity and pore pressure is relatively small due to the smallness of $1-\alpha_{I}$.

more common. We choose constitutive laws for $\tilde{k}$ and $\tilde{p}$ following Smith [1971] and Sharp [1976]:

$$
\begin{aligned}
\tilde{p} & =\ln \left(\phi_{0} / \phi\right)-\left(\phi_{0}-\phi\right), \\
\tilde{k} & =\left(\phi / \phi_{0}\right)^{m},
\end{aligned}
$$

and a typical assumption is that $m$ is large, e.g., $m=8$.

[30] The assumptions in equation (60) are likely to be oversimplified. In particular, we have not made allowance here for the dependence of permeability on clay mineralogy [e.g., Revil et al., 2002], where there appears to be a strong increase (at fixed porosity) of permeability with the illite/ smectite ratio. In our model, this would be represented by allowing $\tilde{k}$ to depend on the smectite and illite volume fractions $\phi_{S}$ and $\phi_{I}$ as well as the porosity $\phi$. While it is not complicated to do so, we have not included this effect here, as we wish to isolate the effect of the diagenetic reaction scheme, and compare the results with those we have obtained previously. It should be noted, however, that the effect of increasing permeability with illite fraction is to offset the decrease with porosity, and this will cause the increased pore pressure gradient found in Figure 1, for example, to become localized in space.

[31] We suppose the sediments lie between a fixed impermeable basement at $z=0$ and a free upper surface at $z=h(t)$ (in conditions of accumulation, this represents the ocean bottom). Suitable boundary conditions are then

$$
\begin{array}{rlrl}
u^{s} & =u^{l}=0 & z=0 \\
\dot{h} & =\dot{m}_{s}+u^{s} & z=h ; \\
\phi & =\phi_{0} & z=h ; \\
\phi_{Y} & =\phi_{Y 0} & & z=h, \quad Y=I, S, F, Q .
\end{array}
$$

These represent conditions at the impermeable basement, the kinematic condition of sediment accumulation at the top surface, prescribed porosity resulting from the condition of zero effective pressure (i.e., pore pressure equals overburden pressure) at the top surface, and prescribed constitution of surface composition of the solid sediments being deposited, respectively.

[32] We define the integrated smectite dissolution

$$
\psi=\int_{0}^{z} R^{*} d z
$$

then integration of the one-dimensional version of equation (40) implies

$$
\phi u^{l}+(1-\phi) u^{s}=-\alpha \psi
$$

where

$$
\alpha=1+\alpha_{F}+\alpha_{Q}-\alpha_{I}-\alpha_{w}
$$

It follows that

$$
u^{s}=-\alpha \psi-\lambda \tilde{k}\left(\frac{\partial \tilde{p}}{\partial z}+1-\phi\right)
$$

and the smectite and illite conservation laws are

$$
\begin{gathered}
\frac{\partial \phi_{S}}{\partial t}=-\frac{\partial}{\partial z}\left(\phi_{S} u^{S}\right)-R^{*}, \\
\frac{\partial \phi_{I}}{\partial t}=-\frac{\partial}{\partial z}\left(\phi_{I} u^{S}\right)+\alpha_{I} R^{*},
\end{gathered}
$$

with equivalent equations for $\phi_{F}$ and $\phi_{Q}$ if $R^{*}$ depends on these also. Without loss of generality we can take $R^{*}=$ $R^{*}\left(\phi_{S}, \phi_{I}\right)$. The evolution of porosity is given by

$$
\frac{\partial \phi}{\partial t}=\frac{\partial}{\partial z}\left[(1-\phi) u^{s}\right]+\left(\alpha+\alpha_{w}\right) R^{*},
$$


and with the choice of constitutive law in equation (60) for $\tilde{p}, u^{s}$ is given from equation (65) by

$$
u^{s}=-\alpha \psi-\frac{\lambda(1-\phi) \tilde{k}}{\phi}\left(\frac{\partial \phi}{\partial z}-\phi\right)
$$

[33] Suitable boundary conditions are then as in equation (61) (the condition on $u$ is redundant, as it has already been used in obtaining equation (63). The kinematic condition determines $h$, and the conditions on $\phi$ at $z=h$ and on $u^{S}$ at $z=0$ are suitable boundary conditions for the parabolic equation (68) for $\phi$. Equations (66) and (67) are then hyperbolic equations for the solid fractions, and the single boundary conditions for $\phi_{S}$, etc., are appropriate for their solution, since the characteristics move downward relative to $z=h$ if $\dot{m}_{s}>0$ as we assume here. These observations also color the numerical method we use. Essentially, a diffusive solver is used to step forward $\phi$ assuming $R^{*}$ is known; then $\phi_{S}$ and $\phi_{I}$ are stepped forward with a hyperbolic solver, and the stepping procedure is iterated toward convergence.

[34] In sections 4.1-4.3, we study the behavior of the solutions of this model in the particular limits of slow and fast compaction. Before doing so, however, we reconsider how the model reaction scheme can be compared to the firstand second-order schemes (22) and (23). The first point to notice is that the application of the weak solubility (or solid density) asymptotics implies that the second-order reaction is essentially equivalent to the first-order scheme (22). If the derivation of the model (20) is followed through for the firstorder scheme, we find that the equations for $\phi_{S}, \phi_{I}$ and $\phi$ are the same, except that the right hand side of the $\phi_{I}$ equation is $M_{I} r_{1} / \rho_{I}$. However, the weak solubility limit implies that $r_{1}=$ $f r_{3}$, and thus we regain the model in equations (66)-(69), the only difference being in the definitions of $R^{*}$ and $\alpha_{Y}$. For a first-order reaction, equation (53) would be appropriate, and the absence of quartz or feldspar reaction (even if the minerals are present as inert species) is simply effected in the model by putting $\alpha_{F}=\alpha_{Q}=0$ : whence we would take $\alpha+\alpha_{w}=1-\alpha_{I}-\alpha_{w}$ in equation (68).

\subsection{Reaction Rates}

[35] The temperature-dependent reaction rates $\mathcal{R}_{i}$ are assumed to be exponentials of the form (see discussion after equation (49))

$$
\mathcal{R}_{i}=\exp \left[\lambda_{i}\left(T-T_{i}\right)\right]
$$

in our simulations we suppose that the temperature increases linearly with depth, thus (dimensionally)

$$
T=T_{0}+G(h-z),
$$

where $G$ is the geothermal temperature gradient and $T_{0}$ is the surface temperature. We define the dimensionless temperature $\Theta$ by

$$
T=T_{0}+G d \Theta
$$

so that the present assumption of a linear temperature gradient gives, dimensionlessly,

$$
\Theta=h-z
$$

and the reaction rates can be written as

$$
\mathcal{R}_{i}=\exp \left[\beta_{i}\left(\Theta-\Theta_{i}\right)\right]
$$

where

$$
\beta_{i}=\lambda_{i} G d, \quad \Theta_{i}=\frac{T_{i}-T_{0}}{G d}
$$

Typical values of the parameters $\beta_{i}$ and $\Theta_{i}$ are $\gtrsim O(1)$ and are used in our numerical illustrations below. More specifically, following the discussion after equation (51), we use representative values $T_{i}-T_{0} \sim 100 \mathrm{~K}, \lambda_{i} \sim 0.07 \mathrm{~K}^{-1}$, $d \sim 1000 \mathrm{~m}$, and the geothermal temperature gradient is typically $25 \mathrm{~K} \mathrm{~km}^{-1}$ [Turcotte and Oxburgh, 1982, p. 53]. With these values, $\beta_{i}=1.75, \Theta_{i}=4$. Observed depths of diagenetic windows in kilometers suggest corresponding ranges of $\Theta_{i}$ should in fact be around 1.5-3. Similar variation of $\beta_{i}$ can be inferred. The parameter $\Theta_{i}$ represents the dimensionless depth where the reaction is "switched on," and the size of $\beta_{i}$ represents the sharpness of the window.

\subsection{Slow Compaction, $\lambda \ll 1$}

[36] When $\lambda \ll 1$, the basic mechanical compaction model (i.e., with no diagenesis) has a compacting basal boundary layer, above which the porosity is essentially constant, and the pore pressure is close to lithostatic, and thus much higher than the equilibrium hydrostatic pressure. In this situation, the effect of diagenesis is to release extra pore water, and this causes the porosity to be increased above its initial value in the diagenetic window, as a result of which the effective pressure becomes negative. This is illustrated for the first-order reaction (53) in Figure 2. A zone of overpressure forms above the diagenetic window, and extends all the way to the surface.

[37] Negative effective pressures are, of course, unphysical, and their occurrence indicates the formation of hydrofracture in the uncemented sediments. A natural hypothesis is then that the effective permeability adjusts itself in order to maintain the effective pressure just at zero in such regions. Figure 3 shows the solution of the model with this assumption. The model equations are the same, but if $\phi$ is computed to be greater than $\phi_{0}$ anywhere, then it is set equal to $\phi_{0}$ there, and equations (67) and (69) are used to compute the effective permeability. We see that a hydrofractured region with a tenfold increase in effective permeability lies above the diagenetic window.

[38] A feature of these results is the relatively well defined diagenetic window between normalized heights $Z=0.2$ and $Z=0.5$. The relative sharpness of this window is associated with the size of the exponent in the reaction rate law (74), and it is possible to obtain analytic solutions in the limit as $\beta \rightarrow \infty$ [Yang, 1998], using "high activation energy" asymptotics. We omit analytic details but illustrate the effect of increasing values of $\beta$ in Figure 4, where we 

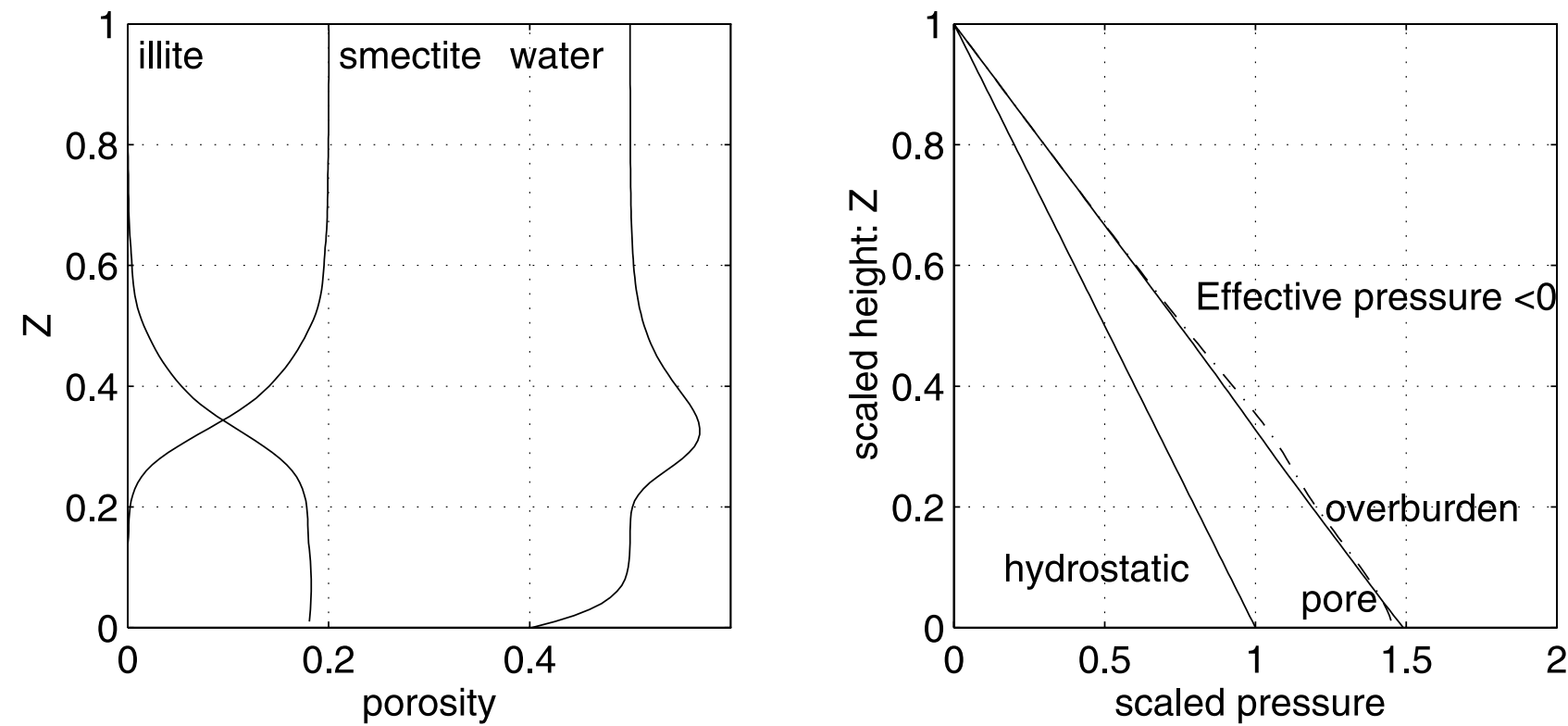

figure 2. First-order reaction kinetics using reaction (22) with $n=2, \beta_{S I}=2.3$, and $\Theta_{S I}=3$. Snapshot of a slowly compacting basin profile when $\lambda=0.01$ at a time $t=5$. The height variable $Z=z / h(t)$ is scaled to lie between 0 and 1. (left) Reaction window between $Z=0.2$ and $Z=0.5$, and porosity profile ("water"), which has the usual basal compaction layer but also a region of excess porosity in the diagenetic window. (right) This leads to a physically unacceptable negative effective pressure.

also show that the effect of changing $\Theta_{i}$ is simply to shift the reaction zone lower in the burial zone.

\subsection{Composite Reaction Kinetics, $\lambda \ll 1$}

[39] We omit presentation of results for the case when $\lambda \ll 1$ for the complex reaction scheme in equation (49), since the pertinent features are unaltered. There is a basal compacting layer, and diagenesis causes an overporous

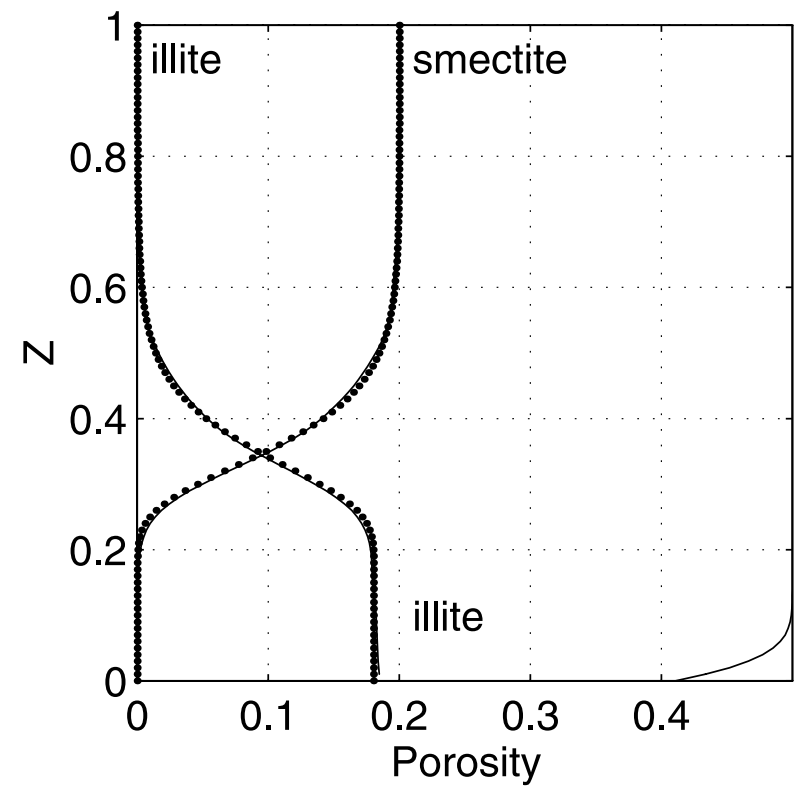

region above the window, which causes hydrofracturing and an associated increase of effective permeability, as we have shown before.

\subsection{Fast Compaction, $\lambda \gg 1$}

[40] When $\lambda \gg 1$, compaction is fast, or sedimentation is slow, and the porosity relaxes rapidly to an equilibrium in which the pore pressure is hydrostatic: hence the effective

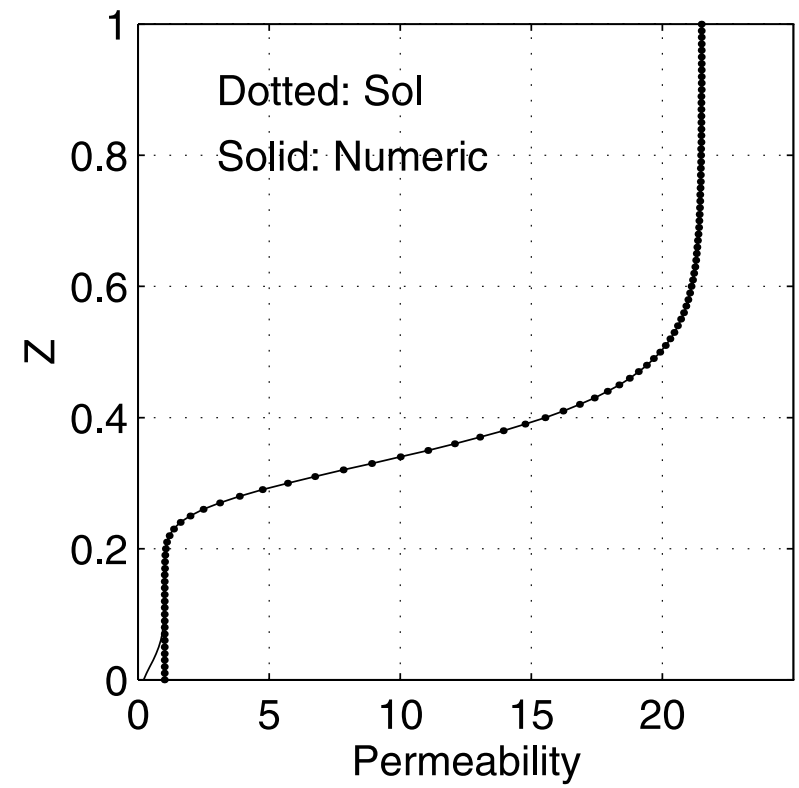

Figure 3. Same calculation as in Figure 2, except that hydrofracturing is allowed to occur in the overporous region and is modeled by constraining the permeability to be such that the minimum effective pressure is zero. The result in Figure 3 (right) is that the effective permeability increases twentyfold, and hydrofracture occurs from the surface down to the diagenetic window. The dotted curves represent an approximate analytic solution [Yang, 1998] which is not presented here. 

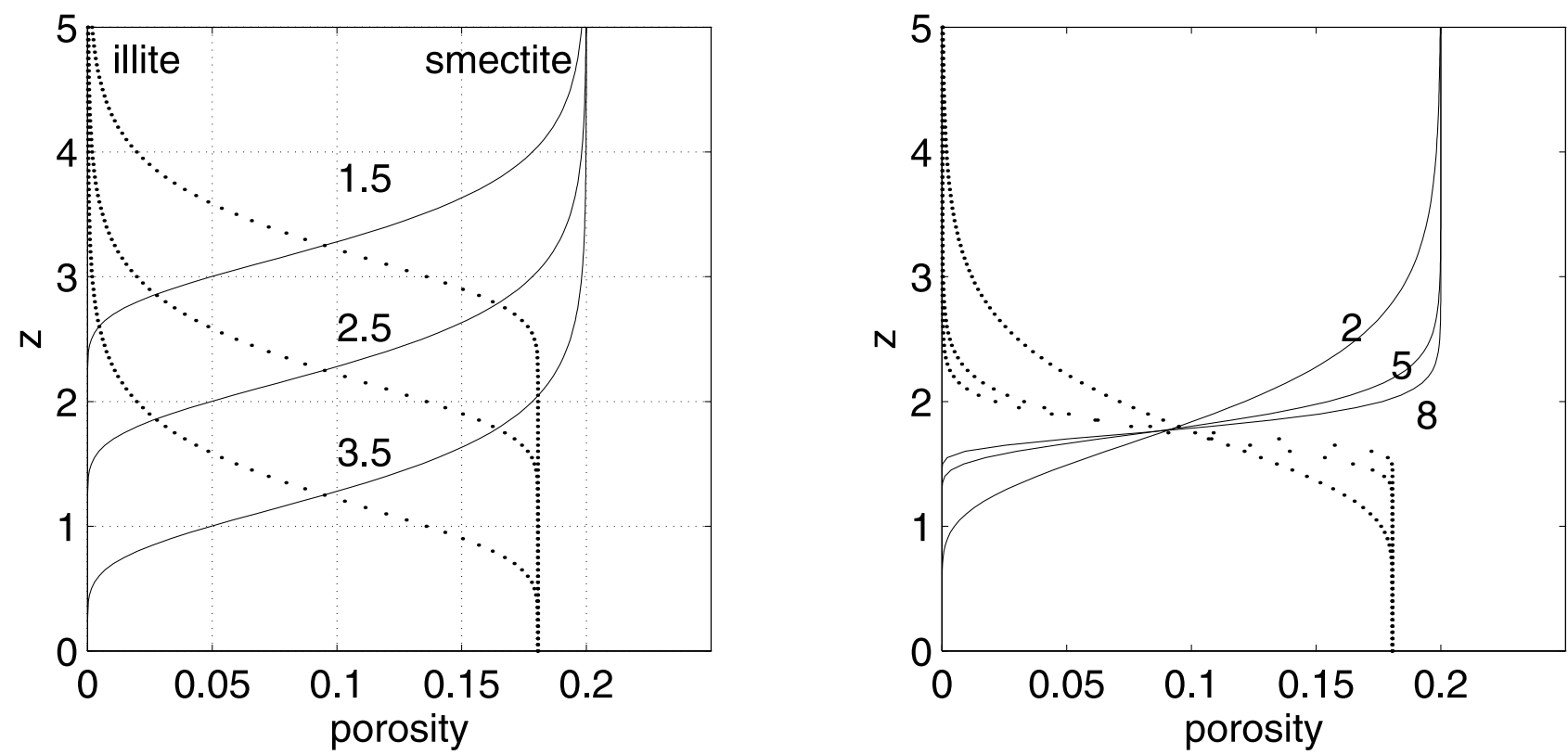

Figure 4. Effect of changing the first-order reaction parameters (left) $\Theta_{S I}$ and (right) $\beta_{S I}$ in equation (74). Clearly, $\Theta_{S I}$ affects the depth of the diagenetic window while increasing $\beta_{S I}$ makes it thinner. The profiles are of smectite and illite with dimensionless height $z$ above the basement, at $t=5$ with $\lambda=0.01$ and $n=2$. The value of $\beta_{S I}$ is 2.3 in Figure 4 (left), and the value of $\Theta_{S I}$ is 3 in Figure 4 (right).

pressure increases with depth, and thus the porosity decreases with depth. However, because the permeability decreases rapidly with the porosity, the high permeability assumption becomes invalid at a critical depth (dimensionlessly, this is denoted by $\Pi=(\ln \lambda) / \mathrm{m}$, and below this, compaction is slow and the pore pressure gradient becomes lithostatic. Figure 1 shows this situation, and also shows the effect of diagenesis. It can be seen that the diagenetic window lies below the transition to low permeability, and this is generally the case. With the temperature $\Theta=h-z$, the diagenetic window begins at depth $\Theta_{i}$, and typical values of this are greater than $\Pi$; for example, $\Pi \approx 0.58$ for $m=8, \lambda=100$.

[41] We see from Figure 1 that diagenesis has little effect on the porosity, or on the pore pressure. The reason for this lies in the amount of dewatering which occurs; this is measured by the value of $\alpha_{I}$, which is the ratio of the molar densities of smectite and illite. Its value is about 0.86 , so that $1-\alpha_{I} \approx 0.14$ and is small. It is in fact possible to derive analytic approximations to the model in this case, and specifically if we assume $1-\alpha_{I} \sim 1 / m \sim \beta_{S I}$; Figure 5 shows the comparison of the theoretical results (Yang 1998) with those obtained numerically. The agreement is reasonable (given that $\beta_{S I}=2.3$ !), and clearly improves for higher values of $\beta_{S I}$, as indicated in Figure 6. More specifically, Fowler and Yang [1998] and Yang [1998] show that below the transition region, $\phi$ is given by

$$
\phi=\phi^{*} \exp \left[\frac{1}{m}(-\ln m+\Psi)\right]
$$

where $\Psi=O(1)$. This applies both if diagenesis occurs and if not, and inspection of equation (6.76) of Yang [1998] shows that the effect of diagenesis is to alter $\Psi$ by $O\left\{\beta_{S I} / m\right.$, $\left.m\left(1-\alpha_{I}\right)\right\}$, which is $O(1)$ for the values we use. Thus the relative variation of $\phi$ due to diagenesis is $O(1 / m)$, consistent with the small variation we see in Figure 1.

\subsection{Composite Reaction Kinetics, $\lambda \gg 1$}

[42] Figure 7 shows a comparison of the first-order kinetic solution with that derived from the composite

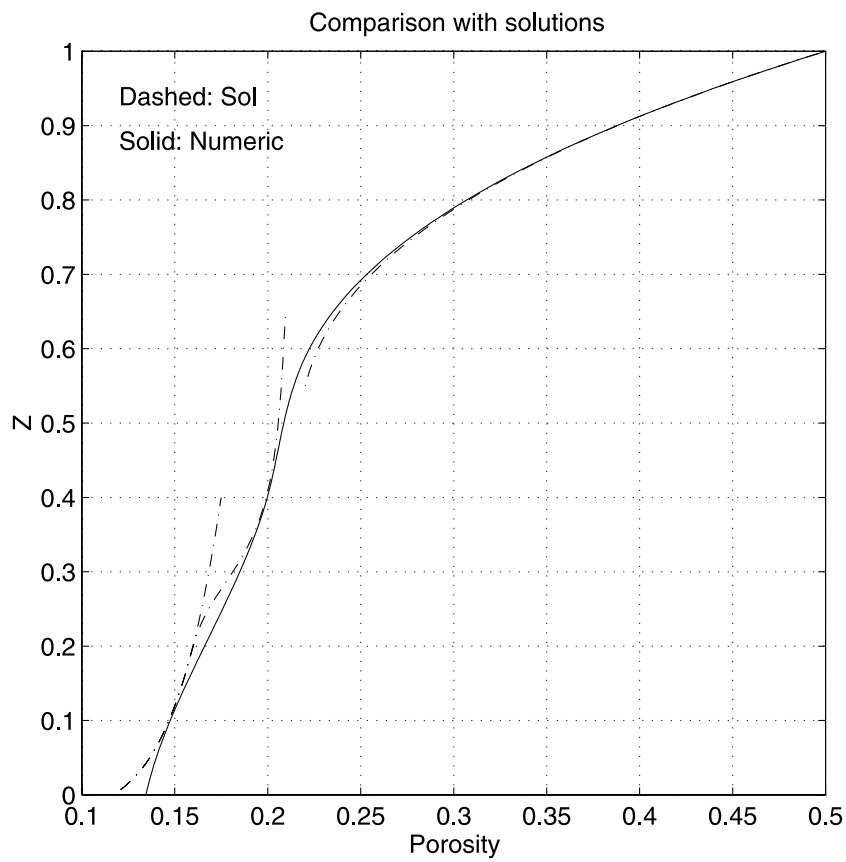

Figure 5. Comparison of theoretical results from Yang [1998], but not presented in the text, with numerical results of the first-order reaction kinetics, when $\lambda=100, n=2, \alpha_{I}=$ $0.86, \alpha_{w}=0.47, \beta_{S I}=2.3, \Theta_{S I}=3$ at time $t=5$. 


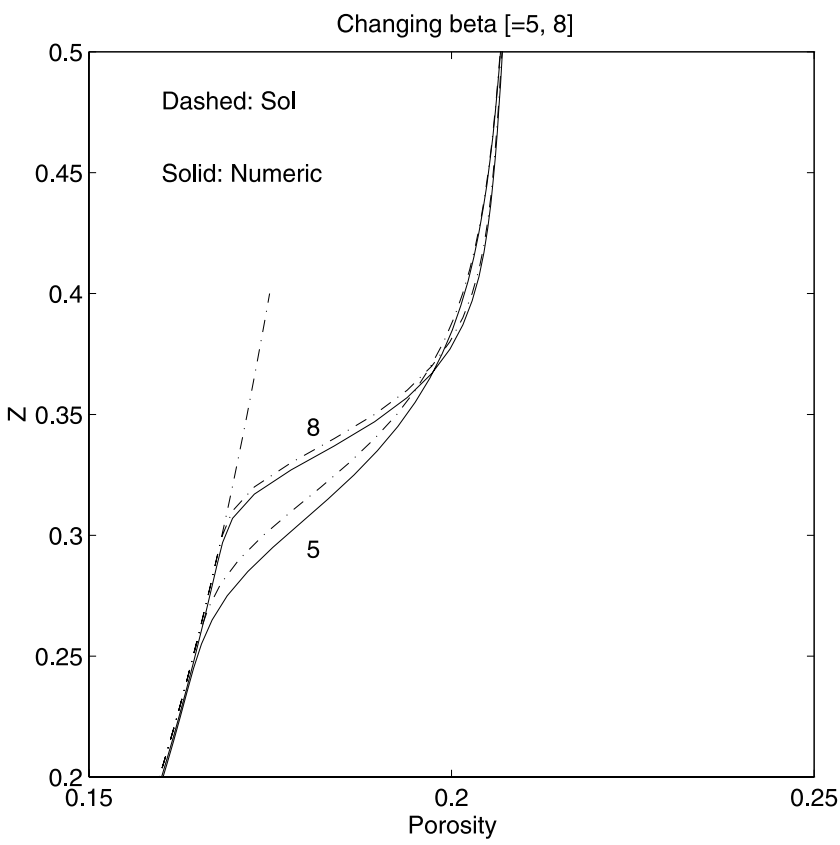

Figure 6. Similar to Figure 5 but a closeup of the diagenetic window with higher values of $\beta_{S I}$, when the approximation improves in accuracy.

precipitation/dissolution scheme (equation (49)), when compaction is fast $(\lambda \ll 1)$. We omit illustration in slow compaction, since in that case the compactive basal boundary layer occurs whatever the reaction scheme, and any reaction leads to the hydrofracturing as before. Figure 8 shows the corresponding pressure profiles. If the kinetic parameters $\beta_{S D}$ and $\beta_{I P}$ are equal and $\Theta_{S D}$ and $\Theta_{I P}$ are equal, then equation (49) is exactly equivalent to reaction (22). In

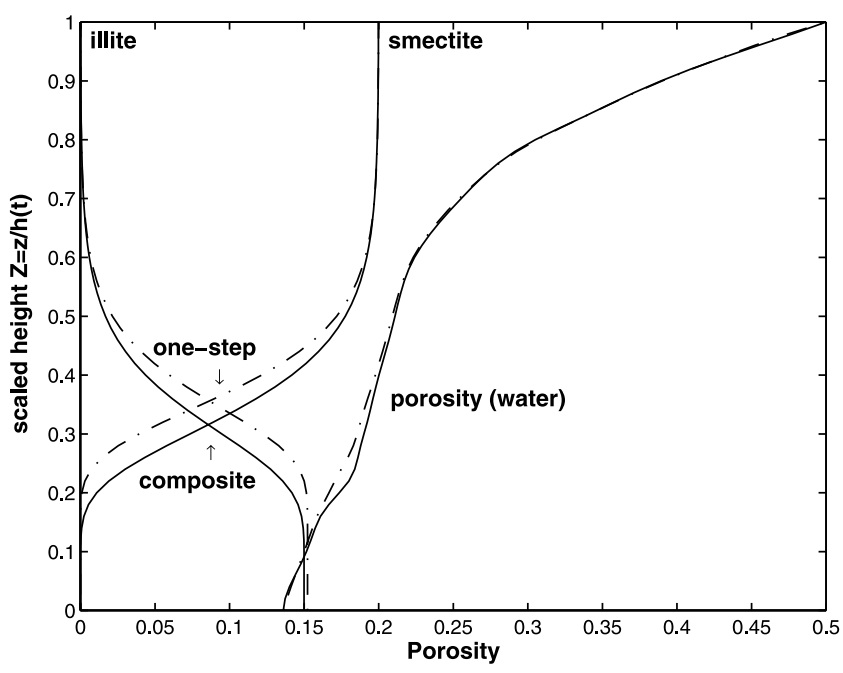

Figure 7. A snapshot comparison of the first-order kinetic reaction (22), using $\lambda=100, n=2, \alpha_{I}=0.86, \alpha_{w}=0.47, \beta_{S I}=$ 2.3, $\Theta_{S I}=3$ at time $t=5$, with the multistep reaction kinetics described by equation (49), with the same parameter values, except that $f=2, \Theta_{S D}=\Theta_{I P}=3, \beta_{S D}=2.3$, and $\beta_{I P}=2.5$. The reaction window is slightly deeper, and the porosity increase is consequently slightly enhanced.

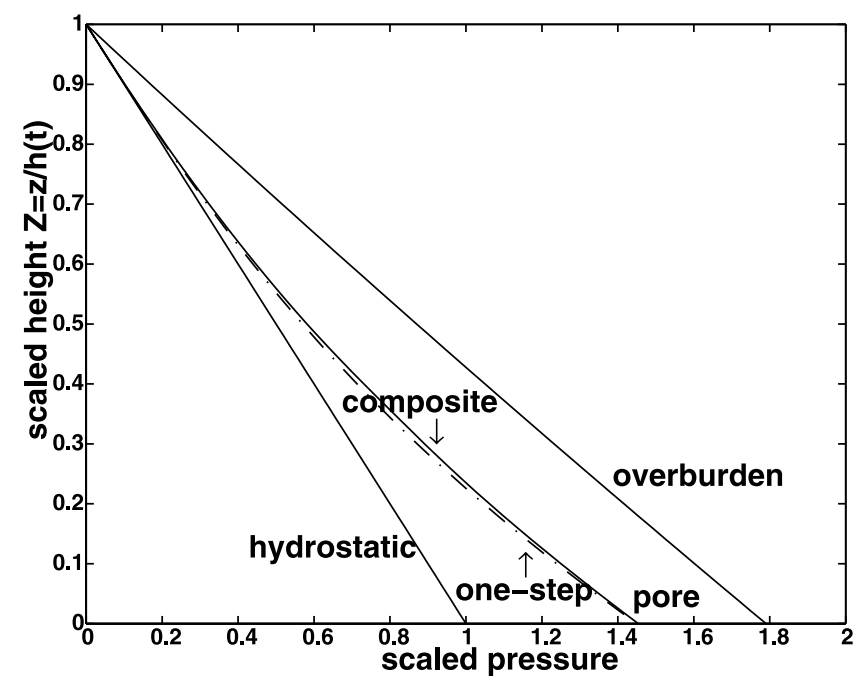

Figure 8. Pressure profiles corresponding to the snapshot in Figure 7. There is little effect of which reaction scheme is used.

practice, the values of these reaction parameters are not likely to be too dissimilar for smectite dissolution and illite precipitation, and this is consistent with the comparability of activation energies and rate constants which are generally reported for water-rock interactions (e.g., Lasaga, 1984), and also with the observation of narrow reaction windows in which both smectite dissolution and illite precipitation take place [Abercrombie et al., 1994]. If this is the case, there is, in fact, little difference between precipitation/dissolution and first-order reaction. Clearly, the difference would be exacerbated if the $\beta$ and $\Theta$ parameters were less comparable.

[43] In Figure 9, we repeat the exercise of Figure 4, in examining the effects of changing either $\Theta_{S D}$ or $\Theta_{I P}$. Clearly, the effects are similar, except that the change of reaction depth with $\Theta_{i}$ is nonlinear, reflecting the bipartite control of the two parameters on the reaction rate, as indicated by equation (49).

\section{Conclusions}

[44] Our principal aim in this paper has been to consider a realistic model of the smectite to illite transformation process, and to show how the corresponding model set of reaction transport equations is related to models derived using simpler kinetics. In particular, we have considered a detailed composite reaction scheme involving dissolution of smectite and precipitation of illite via the intermediary dissolution of potassium feldspar. The kinetics of precipitation and dissolution are very different from mass action derived rate laws, but we have shown that for this particular composite precipitation/dissolution scheme (and, we infer, most probably for more general ones) the application of weak solubility (or, solid density) asymptotics based on the extremely small molar concentrations of aqueous saturated mineral solutions leads to effective reaction equations which are in fact very similar to a model based on first-order kinetics. The primary distinction is that where a first-order model naturally leads to a reaction rate (for smectite dissolution) of $R^{*} \propto \phi_{S}$, the composite reaction rate is a 

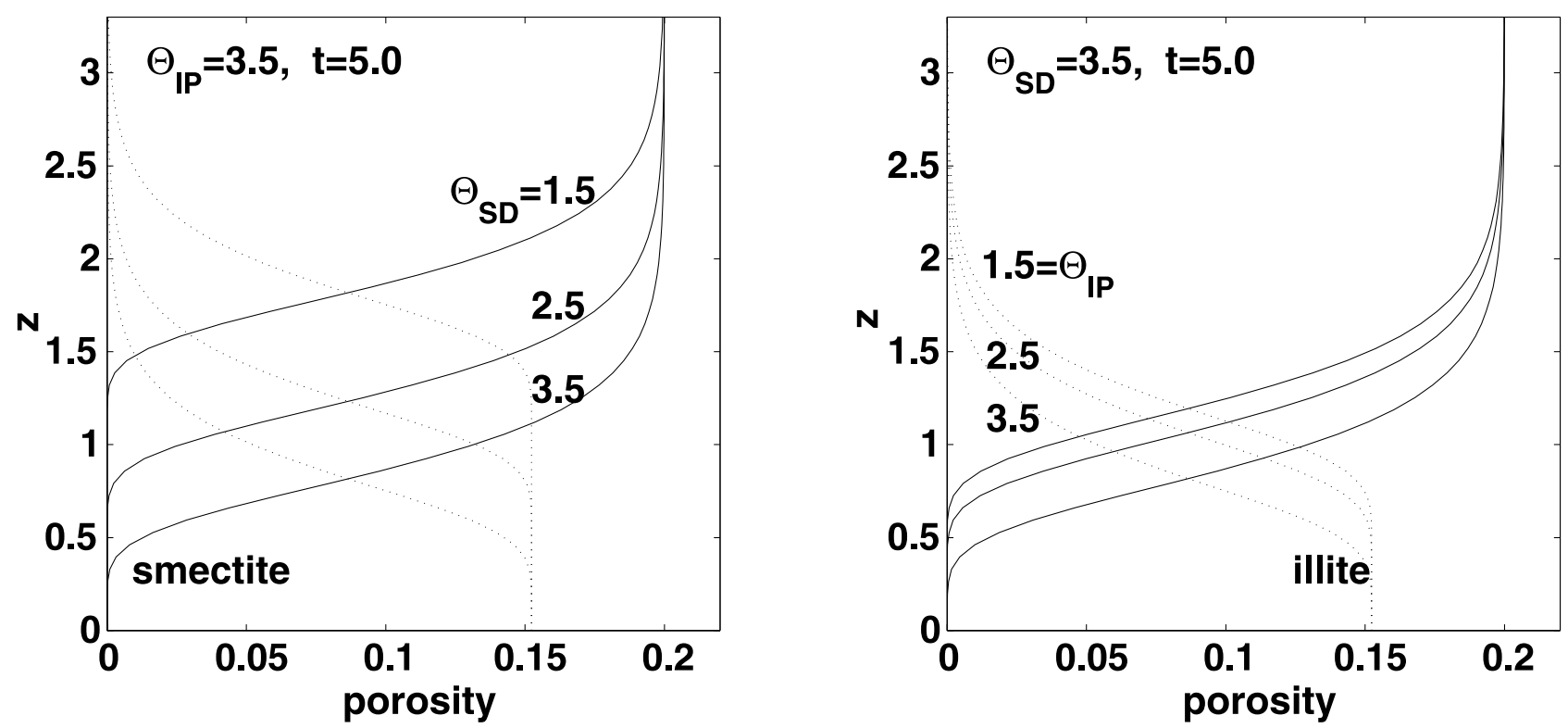

Figure 9. Effects of changing $\Theta_{i}$ on the depth of the reaction window. Snapshots are shown at $t=5$. The parameters are as in Figure 7, except as indicated on the diagrams. This figure can be compared to Figure 4, but note that $\lambda=0.01$ in Figure 4 and thus $\dot{h} \approx 1$, whereas $\lambda=100$ here and $\dot{h} \approx 0.66$, hence the difference in vertical scale.

function of all the solid mineral reactive constituents, and this leads to certain differences in the consequent diagenetic porosity profiles. The weak solubility limit is a very powerful way of producing useful rate equations from very complicated reaction schemes.

[45] We have used the derived reaction model to derive porosity and pore pressure profiles in the limits of fast and slow compaction, and for both the first-order kinetic scheme and an approximate form of the feldspar mediated precipitation/dissolution scheme. When $\lambda \ll 1$, i.e., compaction is slow (sedimentation is fast) then in the absence of diagenesis, pore pressures are almost lithostatic everywhere except near a boundary layer at the basement, and the effect of the extra pore water release due to diagenesis is to cause hydrofracturing to occur. We infer that in this case the effective permeability increases to allow effective pressure to be reduced to zero, and this is a predictive mechanism whereby fractures in sedimentary rocks may be explained.

[46] In the more interesting and probably common situation where compaction is fast, i.e., sedimentation is slow $(\lambda \gg 1)$, the diagenetic window will typically lie below the equilibrium compaction region, where the pore pressure is hydrostatic. Below this, compaction is slow and the pore pressure gradient becomes approximately lithostatic (see Figure 1); however, the effect of diagenesis is fairly small, and this can be ascribed to the relatively small quantity of pore water release, mathematically signaled by the closeness of $\alpha_{I}$ to one. Thus, at least within the confines of the model studied here, diagenesis is not a primary agent for excess pore pressure.

[47] It should be noted that the result in Figure 1 assumes a uniformly decreasing permeability with compaction, i.e., with decreasing porosity. The counteracting effect of permeability increase with illite fraction [Revil et al., 2002] will tend to localize the region of high pore pressure gradient, as found in pressurized seals [Hunt, 1990].

[48] The use of the weak solubility limit allows us to reduce a quite general precipitation/dissolution scheme to one involving two species, the source (smectite) and the product (illite), with a reaction rate which is in general a complicated function of both their concentrations. When source dissolution and product precipitation are slow and thus rate limiting, then the reaction rate is equation (49), and indicates that precipitation and dissolution effectively occur serially (by analogy to two resistors in series). Furthermore, if the reaction parameters $\beta$ and $\Theta$ for the two processes are similar (as here), then in fact the overall scheme is essentially the same as the simpler first-order reaction scheme.

\section{References}

Aagaard, P., and H. Helgeson, Activity/composition relations among silicates and aqueous solutions: II. Chemical and thermodynamic consequences of ideal mixing of atoms on homological sites in montmorillonites, illites, and mixed-layer clays, Clays Clay Miner., 31, 207-217, 1983.

Abercrombie, H. J., I. E. Hutcheon, J. D. Bloch, and P. de Caritat, Silica activity and the smectite-illite reaction, Geology, 22, 539-542, 1994

Aharonov, E., M. Spiegelman, and P. Kelemen, Three-dimensional flow and reaction in porous media: Implications for the Earth's mantle and sedimentary basins, J. Geophys. Res., 102, 14,821-14,833, 1997.

Audet, D. M., and A. C. Fowler, A mathematical model for compaction in sedimentary basins, Geophys. J. Int., 110, 577-590, 1992.

Bjørlykke, K., and P. K. Egeberg, Quartz cementation in sedimentary basins, AAPG Bull., 77, 1538-1548, 1993.

Busenberg, E., and C. V. Clemency, The dissolution kinetics of feldspar at $25^{\circ} \mathrm{C}$ and $1 \mathrm{~atm} \mathrm{CO}_{2}$ partial pressure, Geochim. Cosmochim. Acta, 40, $41-49,1976$

Eberl, D., and J. Hower, Kinetics of illite formation, Geol. Soc. Am. Bull., $87,1326-1330,1976$

Fowler, A. C., and X.-S. Yang, Fast and slow compaction in sedimentary basins, SIAM J. Appl. Math., 59, 365-385, 1998.

Fowler, A. C., and X.-S. Yang, Pressure solution and viscous compaction in sedimentary basins, J. Geophys. Res., 104, 12,989-12,997, 1999. 
Frank-Kamenetskii, D. A., Diffusion and Heat Exchange in Chemical Kinetics, Princeton Univ. Press, Princeton, N. J., 1955.

Freed, R. L., and D. R. Peacor, Geopressured shale and sealing effect of smectite to illite transition, AAPG Bull., 73, 1223-1232, 1989.

Gautier, J. M., E. H. Oelkers, and J. Schott, Experimental study of $\mathrm{K}$-feldspar dissolution rates as a function of chemical affinity at $150^{\circ} \mathrm{C}$ and $\mathrm{pH}$ 9, Geochim. Cosmochim. Acta, 58, 4549-4560, 1994.

Krauskopf, K. B., and D. K. Bird, Introduction to Geochemistry, 3rd ed., McGraw-Hill, New York, 1995.

Hower, J., E. V. Eslinger, M. E. Hower, and E. A. Perry, Mechanism of burial metamorphism of argillaceous sediment: 1 . Mineralogical and chemical evidence, Geol. Soc. Am. Bull., 87, 725-737, 1976.

Huang, W.-L., J. M. Longo, and D. R. Pevear, An experimentally derived kinetic model for smectite-to-illite conversion and its use as a geothermometer, Clays Clay Miner., 41, 162-177, 1993.

Hunt, J. M., Generation and migration of petroleum from abnormally pressured fluid compartments, AAPG Bull., 74, 1-12, 1990.

Lasaga, A. C., Rate laws of chemical reactions, in Kinetics of Geochemical Processes, Rev. Mineral., vol. 8, edited by A. C. Lasaga and R. J. Kirkpatrick, pp. 1-66, Mineral. Soc. of Am., Washington, D. C., 1981.

Lasaga, A. C., Chemical kinetics of water-rock interactions, J. Geophys. Res., 89, 4009-4025, 1984.

Lasaga, A. C., J. M. Soler, J. Ganor, T. E. Burch, and K. L. Nagy, Chemical weathering rate laws and global geochemical cycles, Geochim. Cosmochim. Acta, 58, 2361-2386, 1994.

Nielsen, A. E., The Kinetics of Precipitation, Macmillan, Old Tappan, N. J., 1964.

Ortoleva, P., Geochemical Self-Organisation, Oxford Univ. Press, New York, 1994

Pearson, M. J., and J. S. Small, Illite-smectite diagenesis and palaeotemperatures in North Sea Quaternary to Mesozoic shale sequences, Clay Miner., 23, 109-132, 1988 .

Revil, A., D. Grauls, and O. Brevart, Mechanical compaction of sand/clay mixtures, J. Geophys. Res., 107(B11), 2293, doi:10.1029/2001JB000318, 2002
Rimstidt, J. D., Gangue mineral transport and deposition, in Geochemistry of Hydrothermal Ore Deposits, 3rd ed., edited by H. L. Barnes, pp. 487515, John Wiley, Hoboken, N. J., 1997.

Rimstidt, J. D., and H. L. Barnes, The kinetics of silica-water reactions, Geochim. Cosmochim. Acta, 44, 1683-1699, 1980.

Sass, B. M., P. E. Rosenberg, and J. A. Kittrick, The stability of illite/ smectite during diagenesis: An experimental study, Geochim. Cosmochim. Acta, 51, 2103-2115, 1987.

Sharp, J. M., Momentum and energy balance equations for compacting sediments, Math. Geol., 8, 305-332, 1976.

Smith, J. E., The dynamics of shale compaction and evolution in pore-fluid pressures, Math. Geol., 3, 239-263, 1971.

Steefel, C. I., and A. C. Lasaga, A coupled model for transport of multiple chemical species and kinetic precipitation/dissolution reactions with application to reactive flow in single phase hydrothermal systems, Am. J. Sci., 294, 529-592, 1994.

Turcotte, D. L., and G. Schubert, Geodynamics, John Wiley, Hoboken, N. J., 1982.

Velde, B., and G. Vasseur, Estimation of the diagenetic smectite illite transformation in time-temperature space, Am. Mineral., 77, 967-976, 1992.

Wong, T.-F., S.-C. Ko, and D. L. Olgaard, Generation and maintenance of pore pressure excess in a dehydrating system. 2. Theoretical analysis, J. Geophys. Res., 102, 841-852, 1997.

Yang, X.-S., Mathematical modelling of compaction and diagenesis in sedimentary basins, Ph.D., thesis, Oxford Univ., Oxford, England, 1998.

A. C. Fowler, Mathematical Institute, University of Oxford, 24-29 St Giles', Oxford OX1 3LB, UK. (fowler@maths.ox.ac.uk)

X.-S. Yang, Faculty of Engineering, University of Wales, Swansea, Singleton Park, Swansea SA2 8PP, UK. 